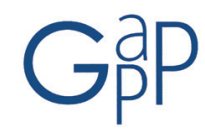

GESTIÓN Y ANÁLISIS DE POLÍTICAS PÚBLICAS, Nueva Época, nº 14 julio-diciembre 2015 ISSN: 1989-8991

DOI: http://dx.doi.org/10.24965/gapp.voi14.10289

\title{
Estrategias de análisis estructural aplicadas a la evaluación de Políticas Públicas
}

\author{
Ignacio Ramos-Vidal|,2 \\ Departamento de Psicología Social (Universidad de Sevilla). \\ Facultad Latinoamericana de Ciencias Sociales (FLACSO-España) \\ ignacioramosvidal@hotmail.com
}

\begin{abstract}
Resumen
Las políticas públicas suelen materializarse a través de programas implementados por diferentes organismos. Cuando son varias agencias las encargadas de llevar a cabo un programa, este conjunto de organizaciones puede adoptar la forma de una red inter-organizativa. El análisis estructural hace posible evaluar este tipo de estructuras en orden a que los resultados de la evaluación puedan incidir positivamente sobre la iniciativa puesta en marcha a través de la red. En este artículo presentamos el potencial del Análisis de Redes Sociales (ARS) para evaluar redes inter-organizativas que ponen en funcionamiento programas de intervención. El manuscrito se divide en cuatro secciones. La primera está dedicada a mostrar antecedentes en los que se emplea el análisis de redes inter-organizativas en la evaluación de programas. En la segunda repasamos los elementos claves para diseñar un análisis estructural, prestando especial atención a los niveles de análisis y a cuestiones relativas al muestreo y al procedimiento de extracción de datos. El tercer bloque se centra en exponer diferentes indicadores que pueden emplearse para evaluar redes interorganizativas. En la última sección reflexionamos sobre el potencial del ARS en la evaluación de políticas públicas y revisamos técnicas emergentes que pueden mejorar los resultados de la evaluación.
\end{abstract}

Palabras clave

Análisis de redes sociales, Coaliciones, Evaluación de programas; Implementación, Políticas públicas, Redes Inter-organizativas.

\section{Structural analysis strategies applied to assess Public Policies}

\section{Abstract}

Public Policies are embodied through programs implemented by different organizations. When several agencies are responsible for carrying out a program, this set of organizations may take the form of an inter-organizational network. Structural analysis make possible to assess this kind of structures so that the results of the evaluation has a positive impact on the outcomes of the initiative developed by the network. In this paper we present the potential of Social Network Analysis (SNA) to assess inter-organizational networks which develops intervention programs. To introduce the key elements to develop a structural evaluation, the paper is divided in four sections. First section is devoted to show antecedents in which inter-organizational network analysis is used to assess programs. In the second section we review the core points to design a structural analysis. The third focuses on expose different measures that can be used to assess inter-organizational networks. In the last section we reflect on the potential of SNA in the evaluation of public policies. Finally, emerging techniques which may improve the outcomes of the evaluation process are reviewed.

Keywords

Coalitions, Implementation, Inter-organizational networks, Program evaluation, Public policies, Social Network Analysis.

1 Esta investigación ha sido financiada parcialmente por la Secretaría de Educación Pública del Gobierno de México, a través de los Fondos PROMEP (Proyecto 13412093).

2 Dirección de correspondencia: Departamento de Psicología Social, Facultad de Psicología: Universidad de Sevilla (España). Calle Camilo José Cela s/n CP (41018), Sevilla (España). Tlfn: + (0034) 954557738. Correo-e: ignacioramosvidal@hotmail.com. 


\section{INTRODUCCIÓN}

En este artículo realizamos una exposición de los principales conceptos, herramientas y estrategias de evaluación estructural que pueden emplearse en el diseño, gestión y análisis de políticas públicas implementadas a través de coaliciones y redes inter-organizativas. Las políticas públicas se llevan a cabo a través de la puesta en marcha de iniciativas que suelen ser desarrolladas por diferentes agencias que trabajan de modo coordinado (Isett et al., 2011). Con frecuencia este conjunto de instituciones adopta la estructura de una red inter-organizativa ${ }^{3}$, una alianza estratégica o una coalición comunitaria. Si bien estos términos pueden responder a diferentes tradiciones, en la práctica comparten puntos en común que los convierten en entidades comparables (Provan, Fish y Sydow, 2007: 480-481). El análisis y la evaluación sistemática de la estructura relacional, derivada de los vínculos que mantienen entre sí los actores que componen la red, constituye una valiosa herramienta para mejorar la coordinación y la efectividad de las iniciativas llevadas a término por el conjunto de organizaciones implicadas en dicho proceso (Jordana, 2009; Lecy, Mergel y Schmitz, 2014).

Con el objetivo de describir las contribuciones específicas del ARS para evaluar redes inter-organizativas que proporcionan servicios públicos, en este artículo emplearemos a título ilustrativo un estudio de caso procedente de una investigación en la que evaluamos una alianza estratégica conformada por organizaciones del sector cultural andaluz (Ramos-Vidal, 2015a; Ramos-Vidal y Maya Jariego, 2013). Para alcanzar este objetivo el artículo se estructura en cuatro bloques. En el primero presentamos la conexión entre el análisis de redes inter-organizativas y la evaluación de políticas públicas. El segundo se centra en describir los puntos clave para diseñar un análisis estructural, concretamente examinamos los niveles de análisis más comunes dentro del ARS, y diversos elementos relacionados con el muestreo y la extracción de datos. El tercer bloque se dedica a examinar los indicadores de estructura y posicionamiento que pueden ser utilizados para evaluar redes inter-organizativas. Por último se incluye un apartado final destinado a sintetizar la información y a exponer las últimas tendencias vinculadas al análisis estructural que pueden aplicarse a la evaluación de políticas públicas.

\section{EL ANÁLISIS DE REDES INTER-ORGANIZATIVAS Y LA EVALUACIÓN DE PROGRAMAS}

En el proceso de diseño, implementación y evaluación de políticas públicas participan múltiples agentes e instituciones que deben trabajar de modo colaborativo para alcanzar los objetivos formulados (Vedung, 2008). Este fenómeno implica que los gestores de las organizaciones, como parte esencial de su trabajo, deben establecer contactos con otros actores para poder cumplir sus metas en relación al programa desarrollado. La gestión de alianzas estratégicas según O’Toole (1997), supone un reto por la complejidad que se asocia a estar inmerso en diferentes estructuras relacionales, complejidad que se ve agravada porque en dichas estructuras suelen participar actores de distinta naturaleza como Administraciones Públicas, clientes, proveedores y organizaciones civiles. En la práctica, formar parte de redes inter-organizativas puede generar importantes beneficios capaces de traducirse en una mayor efectividad de las políticas públicas desarrolladas (Kickert, Klijn y Koppenjan, 1997), en incrementos de la competitividad de las organizaciones que colaboran (Huxham y Vangen, 2005) y en general, en una mejor prestación de servicios públicos (Provan y Milward, 2001).

Asumiendo el inherente componente relacional que caracteriza a la gestión de alianzas estratégicas, las distintas etapas del proceso -desde el diseño hasta la evaluación de las acciones- deben contar con mecanismos específicos de análisis estructural. El conjunto de paradigmas y estrategias analíticas que posibilita la evaluación estructural es el denominado Análisis de Redes Sociales (ARS), que engloba métodos y teorías que analizan los vínculos que se producen entre entidades sociales y las implicaciones que se derivan de estas relaciones (Wasserman y Faust, 1994: 3). Dichas herramientas permiten conocer las propiedades estructurales, es decir, las características que definen la morfología de la red en su conjunto, también denominadas medidas de cohesión (Friedkin, 1981). Mientras que las medidas de centralidad examinan las características de cada uno de los actores que integran la red de forma individual (Freeman, 1979).

En la literatura existe una tradición consolidada que hace un uso extensivo del ARS para examinar la estructura y los factores que inciden en la formación de alianzas estratégicas y coaliciones ${ }^{4}$. El análisis de redes interorganizativas constituye una potente herramienta para evaluar los resultados de programas de intervención que son puestos en marcha mediante este tipo de estructuras (Eisenberg y Swanson, 1996; Provan et al., 2005). Este

3 En la literatura existe cierta superposición epistemológica respecto a los términos red inter-organizativa, alianza estratégica y coalición. En este trabajo asumimos estos términos como equivalentes en la medida en que comparten las cuatro características fundamentales que abordamos en este artículo: (a) que todos los términos hacen referencia a sistemas conformados por diferentes organizaciones, (b) que trabajan de forma colaborativa para desarrollar un programa, (c) que su origen es producto de una demanda social específica, cuya (d) estructura es susceptible de ser evaluada mediante estrategias de ARS.

4 En un artículo previo examinamos las diferentes tradiciones que explican las motivaciones para integrarse en redes inter-organizativas. Para ello véase Ramos-Vidal et al. (2014: 140-142). 
conjunto de técnicas sirven para analizar la estructura, la composición y los roles desempeñados por los diferentes actores implicados, en orden a determinar la configuración estructural capaz de optimizar los resultados de la red. Las coaliciones comunitarias constituyen un claro ejemplo de la creciente utilización de técnicas de análisis estructural para mejorar el funcionamiento organizacional y la prestación de los servicios efectuados a través de este tipo de sistemas (Valente, Chou y Pentz, 2007). Una coalición comunitaria es un conjunto de organizaciones que trabajan en red de forma colaborativa, con el objetivo de implementar iniciativas que mejoren la calidad de vida de la población (Chavis, 2001). Las coaliciones suelen surgir para dar respuesta a una demanda social y en ellas están representadas instituciones públicas, ONGD's, empresas privadas y sujetos individuales entre otros agentes. La función de las coaliciones se focaliza en el desarrollo de un programa de acción específico, por ejemplo en iniciativas estatales diseñadas para reducir el consumo de tabaco (Luke et al., 2010). Sin embargo, en ocasiones la estructura de las coaliciones permanece activa una vez concluido el programa y son utilizadas posteriormente para desarrollar otro tipo de iniciativas aprovechando la estructura creada y los recursos invertidos en el proceso. Desde esta perspectiva, el estudio de la estructura y de la posición que los actores ocupan en la red, constituye un instrumento práctico para evaluar el desarrollo y el cumplimiento de los objetivos del programa que da origen a la coalición (Feinberg, Riggs y Greenberg, 2005). En el siguiente apartado se presentan los elementos clave para diseñar una evaluación estructural.

\section{EL DISEÑO DE UN ANÁLISIS ESTRUCTURAL}

La definición del modelo idóneo de evaluación estructural requiere tomar una serie de decisiones entre las que destacan (a) la selección del nivel de análisis apropiado y la caracterización del tipo de relación objeto de estudio y (b) la delimitación de la muestra y la elección del procedimiento de extracción de información (Knoke y Yang, 2008; Wasserman y Faust, 1994). Emplearemos un estudio de caso basado en una investigación anterior para ilustrar cada una de las etapas del diseño de evaluación estructural. El caso corresponde a una investigación transversal en la que se analizaron múltiples relaciones, en una alianza inter-organizativa conformada por 32 organizaciones en su mayoría privadas, dedicadas a la gestión y promoción de iniciativas culturales en Andalucía (Ramos-Vidal y Maya-Jariego, 2013; Santolaya et al., 2011).

\section{a) Niveles de análisis y contenido de los vínculos}

Una de las primeras decisiones que hay que tomar cuando se realiza un análisis estructural es la selección del nivel de análisis apropiado5. En el ARS habitualmente se consideran tres niveles de análisis, que pueden emplearse individualmente o de modo simultáneo. Estos niveles son inclusivos y se superponen gradualmente. La unidad básica de análisis son los denominados subgrafos, siendo la diada (relaciones entre pares de actores) la más común. El segundo nivel de análisis es el denominado egocéntrico (también denominado red personal), que consiste en aislar las relaciones que un único actor mantiene con el resto de miembros de la red. Por último encontramos el nivel socio-céntrico (también denominada red completa) que engloba a los dos anteriores y que analiza las conexiones entre todos los actores que forman parte de la red. La selección de cada unidad de análisis dependerá de los objetivos y de las características de la investigación.

El análisis diádico es el que cuenta con mayor tradición en el estudio de redes inter-organizativas (i.e., Gulati, 1995), no obstante en los últimos años se aprecia un aumento progresivo de los estudios que emplean el enfoque de redes egocéntricas (Ramos-Vidal, 2015b) y de redes completas (Provan et al., 2007) para este mismo fin. En la tabla 1 examinamos las características de cada nivel, utilizando la representación visual para mostrar cómo se representan las relaciones de una misma organización en cada nivel, en función de distintos tipos de relaciones que denotan vínculos de diferente naturaleza.

En las diferentes representaciones que se observan en la tabla 1 hemos resumido los niveles de análisis que pueden abordarse a través del ARS. En cada fila hemos representado un tipo de relación, al objeto de mostrar que la estructura y la composición de las redes varían substancialmente dependiendo del tipo de relación analizada, con independencia de que la red esté formada por los mismos actores. Los diseños de evaluación estructural deben examinar diferentes vínculos que sean capaces de capturar la complejidad y la multiplicidad inherente a las dinámicas de interacción que caracterizan cada sistema social (Tichy, Tushman y Fombrun, 1979). En el contexto específico de las redes inter-organizativas este requerimiento está especialmente indicado, puesto que las organizaciones establecen diferentes tipos de relaciones con los actores que participan en la estructura, de modo que es preciso analizar diferentes tipos de contactos (p.ej., intercambio de información, solicitud de asesoramiento, ofrecimiento de referencias etc.) y al mismo tiempo es deseable que esas relaciones puedan reflejar diferentes niveles de compromiso y acuerdo (Gulati, 1995).

5 Para una descripción de los niveles de análisis puede consultarse el manual de Wasserman y Faust (1994: 25-26).

GESTIÓN Y ANÁLISIS DE POLÍTICAS PÚBLICAS, Nueva Época, nº 57572015 ISSN: 1989-8991 - DOI: 10.24965/gapp.voi14.10289 
TABLA 1. DESCRIPCIÓN DE LOS PRINCIPALES NIVELES DE ANÁLISIS ESTRUCTURAL

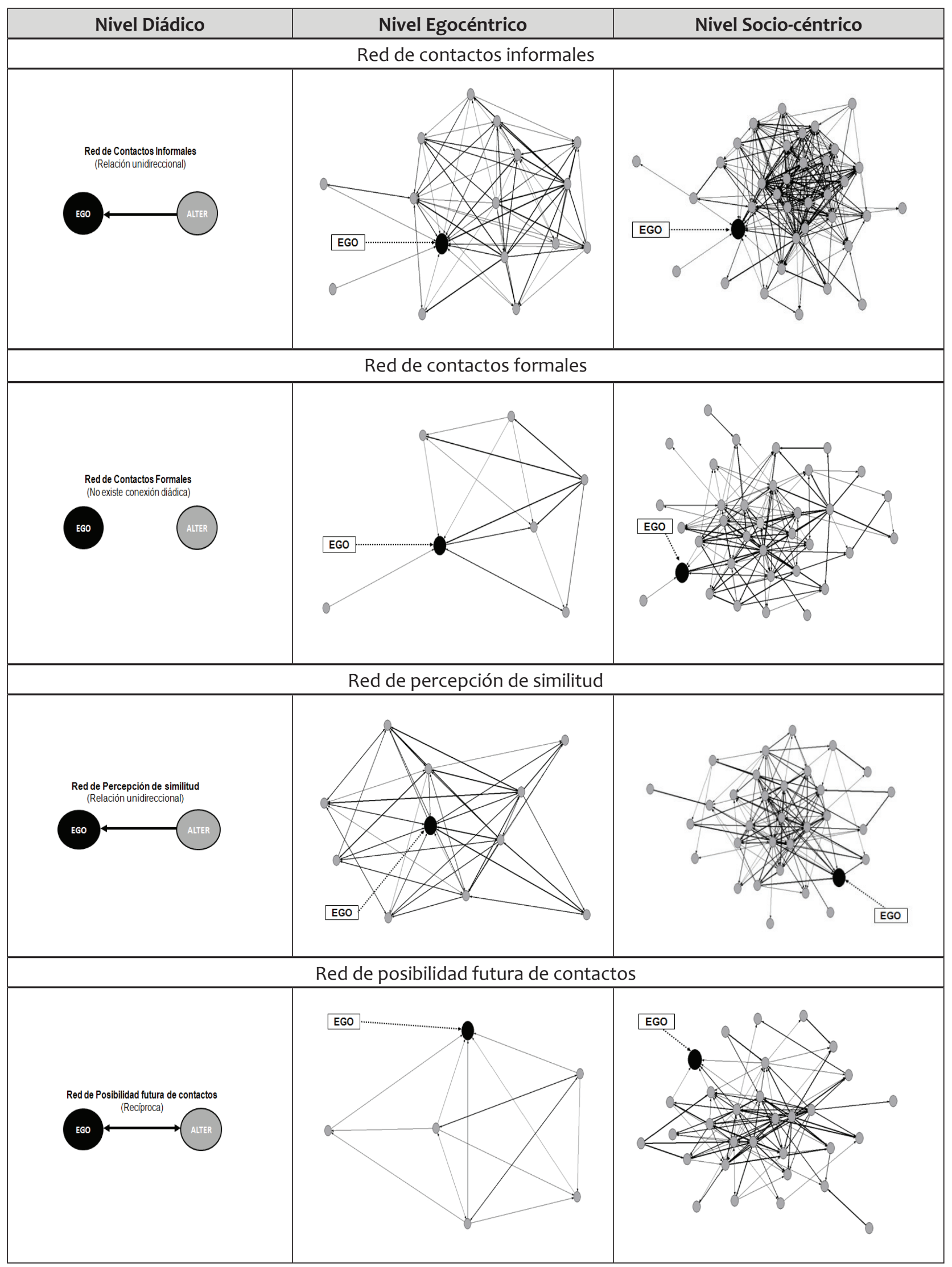

Fuente: Elaboración propia. 
En el estudio que nos sirve a título ilustrativo, analizamos seis tipos de vínculos entre las 32 organizaciones que forman una alianza estratégica que proporciona servicios y productos culturales (Ramos-Vidal y Maya-Jariego, 2013). Los objetivos generales de este estudio fueron (a) poner de relieve la estructura de la red de organizaciones del sector de las artes escénicas en Andalucía, (b) identificar a las organizaciones que ocupan posiciones de influencia dentro de la red y (c) señalar los factores endógenos y exógenos que determinan la ocupación de posiciones de poder. La investigación mostró la existencia de un núcleo altamente cohesivo conformado por organizaciones que desempeñan su actividad en Andalucía Occidental, y al mismo tiempo la existencia de un subgrupo de organizaciones, en su mayoría de la periferia, que se dedican a disciplinas artísticas menos extendidas y que están ubicadas en Andalucía Oriental. Este dato demostró que la proximidad geográfica constituye un factor relevante en la decisión de establecer contactos en este sector. Otro de los hallazgos destacados fue lograr identificar a un pequeño grupo de entidades que articulan el núcleo duro de relaciones, actuando como el eje central de la red. Estas organizaciones pueden considerarse influyentes debido al elevado número de contactos directos que mantienen con una amplia proporción de nodos, aspecto que facilita el acceso a la información y optimiza el proceso de toma de decisiones (Jones, Hesterly y Borgatti, 1997). Finalmente el estudio mostró que la antigüedad en el sector, ostentar una reputación positiva entre los competidores, mantener un nivel de actividad elevado y disponer de un eficiente equipamiento interno, son los factores que más inciden sobre el grado de centralidad de las organizaciones culturales.

De forma concreta, en la investigación examinamos desde relaciones que no exigen ningún tipo de acuerdo previo entre las partes (p.ej., reconocimiento de la organización), hasta relaciones de mayor impacto que requieren altos niveles de confianza y acuerdo (p.ej., participación conjunta en proyectos culturales). Esto nos permite identificar las características del entorno relacional de una organización (red egocéntrica) y de la red completa (red socio-céntrica), en función de un tipo particular de relación y establecer comparaciones entre los distintos tipos de vínculos evaluados, empleando para ello los parámetros de cohesión y de centralidad que veremos en el siguiente bloque. Podemos observar en la primera columna de la tabla 1, la relación que Ego (actor cuyos vínculos particulares están siendo analizados) establece con otro componente de la red (Alter). El análisis diádico constituye la unidad básica de medida, si bien existen otras unidades (tríadas, cliques, n-cliques etc.) que pueden ser analizadas. En el ámbito del análisis de redes inter-organizativas existe una tradición consolidada que emplea la diada para examinar la estructura de relaciones. En este sentido Mizruchi y Marquis (2006: 205) afirman que el criterio para seleccionar el nivel de análisis dependerá entre otros factores del tipo de variable dependiente que se desea evaluar y del número de tipos de relaciones que serán analizados. Los autores afirman que en los casos en los que la variable dependiente es cuantitativa y cuando hay que examinar diferentes tipos de relaciones (por ejemplo intercambio de información y ofrecimiento de referencias), el análisis diádico permite capturar los patrones relacionales de modo eficiente. No obstante, otros autores afirman que el análisis diádico puede ser demasiado básico al estar focalizado en el nivel micro, por lo que es necesario aumentar el foco de la investigación (Rowley, 1997). De otro lado el estudio de las redes egocéntricas (contactos de cada organización) está especialmente aconsejado cuando se trata de conocer la evolución de una organización dentro de la red en su conjunto y en los casos en los que se evalúa el desempeño individual de una entidad en una alianza estratégica. De otro lado el análisis de redes socio-céntricas es el apropiado cuando se trata de capturar el contexto relacional de un conglomerado de organizaciones en su conjunto, mostrando una imagen general del mismo. Por este motivo hemos optado por mostrar los diferentes niveles de análisis que de modo aislado o de forma conjunta, pueden ser empleados cuando utilizamos el ARS para evaluar políticas públicas.

En la segunda columna de la tabla 1, podemos observar la estructura de las redes personales de una organización singular que aparece representada en color negro y mayor tamaño para facilitar su identificación. Como vemos la estructura y composición de la red es diferente en cada uno de los cuatro vínculos explorados. Podemos apreciar cómo la misma organización es muy activa en la red de contactos informales, manteniendo vínculos directos con quince Alteri. Sin embargo esa misma organización presenta una red significativamente más pequeña en el caso de la red de contactos formales, al estar conectada únicamente a seis entidades. Algo parecido sucede cuando examinamos la red personal de percepción de similitud (afinidad) y la red de posibilidad futura de contactos de la misma organización. En este caso, la organización establece hasta diez contactos en la red de percepción de similitud, sin embargo este número se ve reducido a la mitad en la red de posibilidad futura de contactos, mostrando un patrón de relaciones diferencial.

Por último si fijamos la atención en la última columna que se utiliza para representar la estructura completa de relaciones, vemos que sucede un fenómeno idéntico: cada tipo de vínculo ofrece un panorama de conexiones distinto, que a su vez son el reflejo de diferentes dinámicas de interacción en función del tipo de contacto explorado. De modo visual es fácil apreciar que la red de contactos informales es mucho más densa que el resto, en esta red se aprecia un núcleo central altamente cohesionado. Sin embargo tanto en la red de contactos formales, 
como en la red de percepción de similitud la estructura aparece más fragmentada, dejando entrever la existencia de diferentes agrupaciones y un núcleo central menos visible. A continuación efectuamos una aproximación a la etapa del muestreo y a las distintas alternativas que podemos utilizar para extraer la información relacional necesaria para la evaluación estructural de políticas públicas.

\section{b) Muestreo y extracción de datos}

La delimitación de la muestra ${ }^{6}$ es uno de los elementos cruciales a la hora de planificar un diseño de evaluación estructural. Dependiendo de los objetivos de la investigación y del nivel de análisis empleado podemos elegir diferentes opciones. Con frecuencia las redes tienen una estructura cuyos límites están previamente definidos, como por ejemplo el conjunto de países que forma parte del Mercosur o de la Unión Europea, o las organizaciones comunitarias y administraciones públicas que integran una coalición para prevenir el consumo de drogas. En ese contexto, podemos utilizar diseños de investigación de carácter socio-céntrico, en los que se analizan los vínculos que cada actor establece con el resto de componentes de la red. Sin embargo cuando se trata de redes de gran tamaño, por ejemplo cuando nos enfrentamos a grandes coaliciones estatales que cuentan con cientos de miembros, resulta difícil llevar a cabo este tipo de estrategia (Valente et al., 2008). Una de las posibles soluciones consiste en la segmentación en función del contenido de la relación, esto nos permite fragmentar la estructura, posibilitando al mismo tiempo efectuar análisis con unidades más manejables. En el caso de una coalición nacional centrada en actividades de promoción de la salud (i.e., Luke et al., 2010), podría ser viable descomponer la red aislando a los grupos de organizaciones en función del papel que juegan en la red. De este modo podríamos crear subredes a partir de la principal, dependiendo de la función que tiene asignada cada organización, es decir, de si se trata de organizaciones que ofrecen tratamiento, derivan a usuarios, diseñan las intervenciones, implementan las iniciativas o gestionan los recursos implícitos en el desarrollo del programa. Esta alternativa permite trabajar con redes de grandes dimensiones y hace posible mirar dentro de la estructura, acotándola en función del tipo de actividad que desarrollan y del rol que ostentan las organizaciones participantes.

Cuando los límites de la red no están formalmente delimitados o cuando las dimensiones del sistema desaconsejan el desarrollo de un análisis socio-céntrico, podemos emplear diferentes alternativas para capturar el entorno relacional de los actores implicados. Una de las alternativas más recurrentes es el método conocido como muestreo por "bola de nieve" (Biernacki y Waldorf, 1981; Browne, 2005). Este método consiste en identificar a un actor (que constituye el primer eslabón del muestreo), y a partir de los alteri que este actor vaya nominando progresivamente, se construye la estructura de la red. Este tipo de métodos permiten delinear la estructura a partir de la nominación consecutiva de los miembros que se van agregando. Este procedimiento suele emplearse cuando se trabaja con población oculta o difícil de alcanzar, como suele ser el caso de minorías étnicas o personas en riesgo de exclusión social (Atkinson y Flint, 2001; Shelley et al., 1995), no siendo tan frecuente en el ámbito organizacional en el que las relaciones suelen ser visibles o de dominio público (Mizruchi y Marquis, 2006). Un problema relacionado con esta estrategia radica en el momento de culminar el muestreo, esto es, de delimitar la red. Dado que los límites de la red no están formalmente definidos, el procedimiento debería seguir reiterándose hasta que dejasen de aparecer nuevos actores, lo que implicaría que se habría cerrado el círculo de la red. Sin embargo este proceso es inviable en la mayoría de los casos. La alternativa es establecer un punto de saturación, que implica que cuando existe un determinado nivel en el que las nominaciones sucesivas arrojan un alto grado de coincidencia con las anteriores, se puede concluir el muestreo puesto que se habría logrado identificar a los miembros de la red con un margen de error mínimo (Berg, 2006).

Otro elemento clave en el apartado metodológico es la recogida de datos relacionales lo que se conoce como extracción de datos. Existen diferentes alternativas entre las que se incluyen: (a) el uso de cuestionarios, (b) el análisis de datos documentales, (c) la observación y (d) la recolección de datos a partir de redes sociales virtuales.

El empleo de cuestionarios es la forma más común de obtener información relacional. El diseño del instrumento presenta características diferenciales dependiendo de si se trata de un estudio socio-céntrico o egocéntrico. En las investigaciones de tipo socio-céntrico7, en las que conocemos previamente cuáles son los miembros de la red, es necesario crear una matriz en la que en las filas aparecerá un listado con el nombre de los miembros y en las columnas el tipo (o los tipos) de relación que serán analizadas. Posteriormente los datos de todos los actores que han participado se trasladarán a una matriz de adyacencia en alguno de los programas que analizan datos reticulares [Para una descripción de los programas disponibles véase Huisman y Van Duijn (2011)].

6 Para una exposición de las diferentes estrategias de muestreo y cuestiones relacionadas como el error de estimación, la fiabilidad y la recolección de datos longitudinales véase Wasserman y Faust (1994: 43-58).

7 En el Anexo I mostramos un ejemplo de cuestionario socio-céntrico. 
En los estudios egocéntricos ${ }^{8}$ la lógica para extraer la información es distinta, puesto que a priori desconocemos quienes son los integrantes de la red. En estos casos el primer paso es definir las preguntas o generadores de nombres que servirán al entrevistado para identificar y nombrar a los componentes de su red. Por motivos de economía de recursos es frecuente determinar un número máximo de alteri susceptible de ser nominados. En el análisis de redes personales se considera que 30 alteri es un número suficiente para captar la diversidad compositiva de la red (McCarty, 2002). No obstante en el caso de las redes inter-organizativas el umbral puede oscilar notablemente dependiendo del sector. Es de vital importancia caracterizar con detalle el contenido de cada relación objeto de estudio. Esta precisión es igualmente aplicable en el caso de las redes socio-céntricas y egocéntricas.

Una alternativa para recabar información relacional de las organizaciones que participan en la implementación de un programa consiste en el análisis de datos documentales. En estos casos es necesario disponer de un registro o una base de datos en la que queden reflejados los intercambios de información, activos, recursos, personal etc. Esta información suele estar disponible en memorias, documentos de trabajo e informes de gestión, que deben presentar periódicamente los responsables de la implementación del programa para dar cuenta de la evolución del mismo. Esta opción es más fácil de llevar a la práctica debido a que los datos suelen ser accesibles y al mismo tiempo se incurre en menores costes. No obstante la capacidad de generar matrices de adyacencia a partir de información documental limita las posibilidades de extraer otra información relacional de interés, como por ejemplo la que se produce en reuniones informales. Este tipo de información no suele quedar registrada, a diferencia de lo que sucede en el caso de los cuestionarios, en los que el investigador es quien decide el tipo de vínculo que le interesa analizar.

Por otro lado existe la opción de obtener información relacional a partir de la observación. Esta técnica se utiliza con frecuencia en los estudios de carácter etnográfico y en investigaciones que trabajan con población que no puede auto-reportar sus contactos de modo fiable (p.ej., población infantil). Por lo tanto la aplicación de esta estrategia en el ámbito de redes inter-organizativas podría efectuarse en contextos experimentales (p.ej., analizar las interacciones en el transcurso de una reunión de coordinación), pero en el plano aplicado puede ser una decisión poco operativa y de proyección limitada.

Finalmente encontramos una técnica al alza que consiste en la extracción de datos relacionales a partir de la información registrada en redes sociales virtuales como Facebook, Twitter o Linkedln (Ricaurte y Ramos-Vidal, 2015; Russell, 2013). La expansión de las redes sociales virtuales genera una gran cantidad de datos relacionales que pueden ser analizados a través de la disciplina conocida como Data Mining. Tanto las organizaciones como las administraciones públicas participan activamente en redes sociales virtuales, utilizándolas como medio de comunicación, difusión y coordinación. Existen aplicaciones específicas que permiten extraer esta información y seguir procedimientos analíticos similares a los del ARS convencional. Si pretendemos conocer la estructura de relaciones que una organización mantiene a través de Facebook, en primer lugar debemos emplear alguna de las aplicaciones disponibles como por ejemplo Netvizz (Rieder, 2013). A continuación debemos trasladar los datos a alguna de las herramientas diseñadas para visualizar y calcular los parámetros de la red. El software Gephi (Bastian, Heymann, y Jacomy, 2009) permite realizar visualizaciones, cálculos básicos de indicadores e incluye técnicas para detectar subgrupos, sin embargo para un análisis en profundidad es preferible trabajar con programas específicos que analizan redes sociales de gran tamaño como Pajek (De Nooy, Mrvar, y Batagelj, 2011) o JUNG (O'Madadhain, Fisher, Smyth, White, y Boey, 2005). Es necesario considerar que la obtención de datos relacionales por este medio puede estar condicionada por las restricciones y por la política de privacidad que los contactos de la organización hayan establecido en sus respectivos perfiles virtuales, por lo que la efectividad en estos momentos puede ser aún limitada, aunque se prevé un gran desarrollo a corto plazo de este tipo de técnicas.

Una vez expuestas las características del muestreo y los procedimientos de obtención de datos relacionales, en el siguiente apartado se describen los diferentes tipos de métricas que hacen posible comparar y evaluar la estructura de redes sociales.

\section{LOS INDICADORES DE ESTRUCTURA Y POSICIONAMIENTO}

Un punto clave para comprender el papel del análisis estructural en la evaluación de políticas públicas, radica en la posibilidad de monitorizar los resultados de la intervención, examinando (a) las alteraciones en la estructura de la redes inter-organizativa encargadas de la implantación del programa y (b) los cambios en la posición que ocupa cada actor que forma parte de la red (Stufflebeam y Shinkfield, 2007). Encontramos numerosos antecedentes en los que el análisis estructural es utilizado como herramienta de evaluación de programas y políticas públicas. Cuando los estudios son de carácter transversal, los resultados nos permiten tomar una instantánea, que describe las propiedades estructurales de la red en un momento específico, realizando un análisis estático de la colaboración

8 En el Anexo II mostramos un ejemplo de cuestionario para obtener datos egocéntricos.

GESTIÓN Y ANÁLISIS DE POLÍTICAS PÚBLICAS, Nueva Época, nº 61612015 ISSN: 1989-8991 - DOI: 10.24965/gapp.voi14.10289 
entre las organizaciones que componen la red. En esta línea Harris y colaboradores (2008) utilizaron el ARS para evaluar una amplia red inter-organizativa en la que participan diferentes organismos, cuyo objetivo central es la aplicación de un programa de prevención del consumo de tabaco en Estados Unidos. El análisis estructural sirvió como diagnóstico de la estructura, mostrando la existencia de un núcleo central de organizaciones que lideraban el proceso de coordinación de las acciones y la existencia de subgrupos conformados por agencias que presentaban características en común. En una propuesta reciente Buchthal y colaboradores (2013) emplearon el ARS para evaluar las interacciones entre diferentes agencias que colaboran en una red diseñada para promover estilos de vida saludables en la población. Sus hallazgos evidenciaron una segregación espacial entre las organizaciones implicadas en la puesta en práctica del programa y la existencia de una amplia distancia media ${ }^{9}$ entre los diferentes actores, factor que ralentizaba la comunicación y dificultaba las tareas de coordinación.

No obstante, a pesar de valorar la capacidad de los análisis transversales para mostrar el contexto social, coincidimos con el argumento de Abbasi y Kapucu (2012), cuando señalan que el análisis estático logra capturar el entorno relacional en el que se desenvuelven las organizaciones en un momento determinado, sin embargo para comprender los patrones y los efectos que los cambios en la estructura y en la composición de las redes producen en los programas que desarrollan, es necesario efectuar análisis longitudinales capaces de capturar la evolución temporal de las redes inter-organizativas. Desde esta segunda perspectiva, identificamos contribuciones que analizan los cambios en la estructura de la red y los efectos en el desarrollo de los programas. En un trabajo reciente Haines, Godley y Hawe (2011) emplearon el análisis estructural con el objetivo de indagar en los cambios producidos en una red inter-organizativa diseñada para implementar políticas de promoción de la salud. Identificaron que cambios substanciales en las medidas de cohesión de la red completa, se relacionaban con mejoras en la coordinación entre las agencias que participaban en la coalición. Mostraron que aumentos en la densidad global de la red y la reducción del número de actores aislados, se relacionaban con un mejor desempeño de la red inter-organizativa. En otro artículo Ramanadhan et al. (2012) analizan las transformaciones que se produjeron en los cuatro años de funcionamiento de una amplia coalición orientada a la prevención de cáncer que estaba formada por profesionales de la salud, organizaciones comunitarias e instituciones públicas. La información recabada a través del ARS permitió demostrar que los actores que habían incrementado sus conexiones con otras agencias en este período, es decir, aquellas que habían experimentado aumentos en la centralidad de grado, se correspondían con las organizaciones que habían desarrollado más actividades de promoción de la salud, implicándose de forma más activa en la implantación del programa. Estos resultados demuestran que los cambios en la estructura relacional, tienen capacidad para influir de modo directo en el resultado de las iniciativas llevadas a cabo por las coaliciones. En la tabla 2 presentamos una relación de los indicadores de centralidad y cohesión que se emplean con mayor frecuencia para evaluar los cambios en las redes inter-organizativas, capaces de afectar a los resultados de los programas que llevan a cabo.

TABLA 2. CONEXIÓN ENTRE LOS INDICADORES DE ARS Y LA EFECTIVIDAD DE LOS PROGRAMAS

\begin{tabular}{|c|c|c|}
\hline Tipo & Nombre y descripción básica & Antecedentes \\
\hline \multirow[t]{4}{*}{$\begin{array}{l}\text { Cohesión } \\
\text { (estructura } \\
\text { de la red } \\
\text { completa) }\end{array}$} & $\begin{array}{l}\text { Densidad: Proporción de contactos que se produce en una red social, } \\
\text { en comparación con el total de vínculos posibles. Los incrementos en la } \\
\text { densidad suelen reflejar un estrechamiento en la colaboración y mejor } \\
\text { coordinación entre los actores que participan en la red. }\end{array}$ & \multirow{4}{*}{$\begin{aligned}- & \text { Bunger et al. } \\
& (2014) \\
- & \text { Feinberg et al. } \\
& (2005) \\
- & \text { Gulati y Sytch } \\
& (2007) \\
- & \text { Haines et al. (2011) } \\
- & \text { Isett y Provan } \\
& \text { (2005) } \\
- & \text { Luke et al. (2010) } \\
- & \text { Ramanadhan et al } \\
& \text { (2012) } \\
- & \text { Ramos-Vidal y } \\
& \text { Maya-Jariego } \\
& \text { (2013) } \\
- & \text { Valente et al. } \\
& \text { (2008) }\end{aligned}$} \\
\hline & $\begin{array}{l}\text { Actores Aislados: Los cambios estructurales que derivan en un menor número } \\
\text { de nodos aislados suelen reflejar una mejoría en los flujos de comunicación y } \\
\text { en la integración en la coalición que lleva a cabo el programa. }\end{array}$ & \\
\hline & $\begin{array}{l}\text { Reciprocidad: Señala el grado en que los vínculos emitidos son } \\
\text { correspondidos, es decir, la medida en que las relaciones son bidireccionales. } \\
\text { Incrementos en esta medida indican mejor coordinación y aumento en los } \\
\text { flujos de información que tienden a redundar en mayor efectividad. }\end{array}$ & \\
\hline & $\begin{array}{l}\text { Centralización: Este indicador evalúa la medida en que las relaciones se } \\
\text { concentran en un grupo reducido de actores (o grupos de actores). Valores } \\
\text { altos en este indicador suelen ser el reflejo de estructuras disfuncionales, } \\
\text { salvo en las primeras etapas de la coalición en las que es normal que se dé } \\
\text { un alto nivel de concentración de vínculos en pocos actores. }\end{array}$ & \\
\hline
\end{tabular}

9 En este caso empleamos el término distancia desde el punto de vista estructural, no desde la perspectiva geográfica. La distancia estructural en el ARS se mide a través de diferentes indicadores como la Cercanía (Closenness), que analiza los grados de separación que existen entre dos actores o subgrupos de actores. Para una revisión de los indicadores de centralidad véase Freeman (1979). 


\begin{tabular}{|c|c|c|}
\hline Tipo & Nombre y descripción básica & Antecedentes \\
\hline \multirow{3}{*}{$\begin{array}{l}\text { Centralidad }{ }^{10} \\
\text { (Examinan la } \\
\text { posición de } \\
\text { cada actor en } \\
\text { la red) }\end{array}$} & $\begin{array}{l}\text { Centralidad de Grado: Señala las veces que un actor ha nominado } \\
\text { (grados de salida) o ha sido nominado (grados de entrada) por el resto } \\
\text { de actores. Incrementos en este indicador se relacionan con una mejor } \\
\text { integración dentro de la red en función del tipo de vínculo explorado. }\end{array}$ & \multirow{3}{*}{$\begin{array}{l}\text { Abbasi y Kapucu } \\
\text { (2012) } \\
\text { Harris et al. (2012) } \\
\text { Li et al. (2012) } \\
\text { Owen-Smith y Powell } \\
\text { (2004) } \\
\text { Provan et al. (2009) } \\
\text { Wendel et al. (2010) }\end{array}$} \\
\hline & $\begin{array}{l}\text { Intermediación: Muestra las veces que un actor se sitúa en el camino más } \\
\text { corto (geodésico) que existe entre dos actores. Indica la ocupación de } \\
\text { posiciones estratégicas en la estructura y pueden ser el indicador de una } \\
\text { mejor adaptación del actor en el desarrollo del programa. Esta medida } \\
\text { sirve para identificar a los actores que pueden ejercer como difusores de } \\
\text { buenas prácticas al resto de miembros. }\end{array}$ & \\
\hline & $\begin{array}{l}\text { Cercanía: Grado en que un actor se encuentra próximo al resto de } \\
\text { actores que forman parte de la red. Los aumentos en esta medida } \\
\text { indican mejoras en el funcionamiento de la coalición y en el tráfico de } \\
\text { información que fluye a través de la red inter-organizativa. }\end{array}$ & \\
\hline
\end{tabular}

Fuente: Elaboración propia

La información aportada en la tabla 2 indica la intensa conexión que existe entre los indicadores estructurales y de posicionamiento, y el funcionamiento de las redes encargadas de implementar los programas. Si bien no se trata de un listado exhaustivo, puesto que dentro de ambos grupos de medidas existen otros indicadores que son utilizados para examinar este tipo de redes, en la práctica pueden servir de referencia a los gestores de coaliciones, para analizar los cambios estructurales que se van produciendo a medida que se aplica el programa.

En líneas generales las medidas de cohesión nos aportan información relevante sobre el grado de compactación de la red. Inicialmente las coaliciones tienen una estructura formada por un pequeño núcleo central, constituido por actores que desarrollan funciones de coordinación, mientras que el resto ocupa la zona externa de la red y presentan pocas conexiones con los otros integrantes e incluso pueden llegar a estar completamente aislados (Valente et al., 2008). Sin embargo, a medida que la coalición pone en funcionamiento el plan de acción, es de esperar que se produzcan cambios sustantivos en los patrones de interacción y en la estructura del sistema. De este modo es habitual que se registren aumentos en la densidad, en la transitividad y en la reciprocidad, al igual que suele reducirse el número de actores aislados. Estos cambios suelen ser el resultado de mejoras en la comunicación, en la coordinación y de un intercambio de información efectivo entre las agencias participantes. De otro lado una disminución significativa del nivel de centralización global y de algunos indicadores asociados ${ }^{11}$, suele ser un signo de la integración de las organizaciones periféricas en el núcleo central de la red (Haines et al., 2011; Feinberg et al., 2005), dando paso a diseños más equilibrados en relación a la distribución de contactos entre la estructura central y periférica.

En cuanto a los parámetros de centralidad, cabe mencionar que su análisis está especialmente indicado cuando el objetivo reside en evaluar el rol desempeñado por cada organización de forma independiente, o cuando se trata de detectar organizaciones potencialmente influyentes (Ramos-Vidal, 2015b). Por ejemplo si estamos analizando una red de transferencia de información, los incrementos en la centralidad de grados de salida (Outdegree) de un actor, evidencian un aumento de la actividad y de las conexiones con el resto de actores. Mientras que incrementos notables en la centralidad de grados de entrada (Indegree), pueden mostrar una mayor prominencia de la organización y un incremento de la confianza y de la legitimidad manifestada por el resto de actores. En el caso de la intermediación se observa que aquellas organizaciones que experimentan incrementos en este indicador, adquieren posiciones estratégicas que les posibilitan aumentar su capacidad para incidir sobre el resto de actores. Finalmente los aumentos en la cercanía, suponen una reducción de la distancia media que separa a un actor del resto de componentes de la red. Aquellas entidades que cuentan con un alto grado de cercanía, incurren en menores costes para trasladar información y para tener acceso al resto de organizaciones.

Los actores que ocupan posiciones estratégicas que dan acceso a diferentes subgrupos son denominados brókeres. En un estudio reciente Long, Cunningham y Braithwaite (2013) muestran a través de una revisión sistemática, que la identificación de estos actores incide positivamente en (a) la generación de innovaciones, (b) la difusión de conocimiento y en (c) facilitar la confianza y el compromiso entre los miembros de la red. Por

10 Debemos tener en consideración que a partir de los valores agregados de las medidas de centralidad, es posible calcular los valores medios para la red en su conjunto.

11 Existe un indicador denominado Coreness que permite evaluar el grado en que los actores se sitúan en el centro o en la periferia de la red. Para calcular este indicador se utiliza el modelo centro-periferia continuo el cual considera que las redes están compuestas por varios centros, cada uno de los cuales con su correspondiente estructura periférica (Borgatti y Everett, 1999). 
tanto localizar a estos actores a través de las herramientas de ARS, puede ser una estrategia de gran utilidad para mejorar el funcionamiento de la coalición, contando con ellos como aliados necesarios para el desarrollo efectivo del programa. Además de las medidas de centralidad, existen otros mecanismos para detectar actores clave que utilizan técnicas de identificación de subgrupos e indicadores de posicionamiento alternativos (RamosVidal, 2015b).

El posicionamiento de los actores y las propiedades estructurales suelen ser analizadas en base a la distinción dicotómica que diferencia el centro y la periferia de la red. La estructura central está conformada por actores que mantienen múltiples conexiones entre sí y que al mismo establecen vínculos débiles con los actores periféricos, mientras que la periferia se integra por actores que prácticamente no mantienen contactos entre sí, aunque mantienen débiles conexiones con los actores centrales (Borgatti y Everett, 1999: p. 378). La ocupación de posiciones centrales se relaciona con una mayor conectividad y con mayores posibilidades de acceder a fuentes de información y recursos. Por ejemplo Mascia, Cicchetti y Damiani (2013) analizaron una red de transferencia de conocimiento formada por profesionales de la salud, demostrando que el centro de la red estaba ocupado por los profesionales con mayor capacidad para poner en práctica protocolos sanitarios basados en la evidencia empírica. Estos resultados reflejan el potencial que ofrece la evaluación estructural para mejorar la efectividad de los programas.

Una vez descritos los puntos clave que deben tenerse en consideración para aplicar el ARS en el ámbito de la evaluación de políticas públicas, en el apartado final proponemos algunas reflexiones que hagan posible obtener el máximo beneficio de los resultados derivados de un análisis estructural y describimos algunas técnicas que se están desarrollando para fortalecer la evaluación de políticas públicas a través del ARS.

\section{CONCLUSIONES Y ORIENTACIONES DE FUTURO}

El principal objetivo de este artículo ha sido mostrar el potencial que ofrece el ARS para evaluar redes interorganizativas y alianzas estratégicas, que suelen ser los sistemas encargados de implementar programas de acción que constituyen el principal vehículo para el desarrollo de políticas públicas. Para ello hemos ofrecido una introducción a las técnicas y herramientas que hacen posible analizar de forma sistemática la estructura de las redes inter-organizativas y conocer el rol que desempeñan los actores que participan en el plan de acción.

En el plano metodológico hemos visto cómo los gestores de políticas públicas que opten por llevar a la práctica una evaluación estructural, deben tomar una serie de decisiones importantes relativas a la etapa de diseño y a la concepción misma del estudio. Uno de los elementos más relevantes tiene que ver con el nivel de análisis que será evaluado. El análisis diádico es aconsejable cuando se disponen de datos longitudinales y en particular en los casos en los que es preciso analizar diferentes tipos de relaciones. Sin embargo centrar el foco de atención en este nivel, a menudo impide ver más allá del vínculo particular que mantienen las organizaciones que componen la diada y a veces no se tienen en cuenta otros factores que afectan al sistema completo y que pueden incidir en las relaciones que tienen lugar entre cada par de actores (Mizruchi y Marquis, 2006: 205). Este hecho, unido al principio de interdependencia que implica que el posicionamiento de un actor -o de una diada-, se ve influido por el comportamiento del resto de nodos que componen la red (Mitchell, 1969), en cierto modo supone obviar el efecto que otros actores pueden ejercer sobre el comportamiento de los nodos que componen la diada objeto de estudio.

De otro lado el enfoque de redes egocéntricas, si bien no cuenta con tanta tradición en el análisis de contextos inter-organizativos como el anterior, cada vez se utiliza más por su versatilidad a la hora de capturar el contexto relacional de las organizaciones. Este nivel es el preferible cuando el objeto de estudio es evaluar el rol que una organización singular desempeña en la puesta en marcha del programa, pero es necesario definir con claridad los generadores de nombres y el contenido de las relaciones que delimitarán la red de cada organización. Este tipo de análisis debe complementarse con un estudio pormenorizado de las características de cada organización, de su trayectoria dentro de la red y de los factores -internos y externos- que pueden estar incidiendo en la configuración estructural (Ramos-Vidal y Maya-Jariego, 2013).

Finalmente el nivel de análisis socio-céntrico, engloba a los dos anteriores y también está experimentando un desarrollo emergente dentro del análisis de redes inter-organizativas (Provan et al., 2007). Desde nuestro punto de vista el nivel de análisis socio-céntrico constituye un vehículo privilegiado para desarrollar análisis de evaluación estructural por los siguientes factores. En primer lugar, este diseño simplifica notablemente la delimitación de la red, que es una de las decisiones más complejas que se deben tomar en este proceso. En segundo lugar, la realización de un análisis socio-céntrico hace posible capturar diferentes tipos de relaciones que muestran diferentes tipos de estructuras relacionales como vimos en la tabla 1. Esto hace posible trabajar de modo simultáneo con diferentes 
redes, conformadas por las mismas organizaciones y también posibilita agregar las relaciones creando una matriz de contactos a partir de la adición de todas las relaciones exploradas. Finalmente decantarnos por la red completa como unidad de análisis también facilita explorar los vínculos que se producen en unidades inferiores de análisis, es decir, con diadas y con redes egocéntricas. Como vimos en la tabla 1, podemos realizar operaciones sencillas para extraer la red egocéntrica de una organización que forma parte de una red socio-céntrica, y posteriormente calcular medidas de cohesión para comparar ${ }^{12}$ la estructura de las redes de cada organización. También existen técnicas específicas que sirven para efectuar análisis diádicos empleando datos socio-céntricos. Por ejemplo el Procedimiento de Asignación Cuadrática realiza análisis de la red completa utilizando la diada como unidad de referencia. Esta técnica hace posible (a) comparar múltiples redes de modo simultáneo, (b) establecer correlaciones entre los diferentes vínculos e incluso (c) examinar relaciones de dependencia entre varias redes a través de modelos de regresión (Dekker, Krackhardt, y Snijders, 2007; Krackhardt, 1988; Ramos-Vidal, 2015C). Por tanto consideramos que el nivel de análisis socio-céntrico puede ser una opción acertada y versátil capaz de ofrecer una imagen certera de una red inter-organizativa, al mismo tiempo que su elección es compatible con la posibilidad de analizar las relaciones a nivel diádico y egocéntrico.

Las alternativas que hemos ido desgranando a lo largo del texto, podríamos decir que constituyen la perspectiva tradicional dentro de las técnicas de ARS. Sin embargo están surgiendo nuevas tendencias que incluyen robustas herramientas de investigación, que en algunos casos sirven de complemento a las técnicas convencionales y en otros se erigen en nuevos modelos de evaluación estructural. De forma sintética trataremos de ofrecer una semblanza de algunas de estas técnicas entre las que destacan: (i) el análisis de datos longitudinales; (ii) los modelos de simulación basados en agentes; (iii) los sistemas de posicionamiento geográfico y (iv) la combinación de estrategias de investigación cuantitativas y cualitativas (métodos mixtos).

En los últimos años se ha producido un notable desarrollo de programas y técnicas para analizar medidas repetidas de datos relacionales o lo que es lo mismo para analizar datos longitudinales (Robins et al., 2007). Estas herramientas hacen posible conocer no sólo cuál es la estructura de la red en un momento puntual, sino que permiten indagar en la evolución del sistema, estableciendo relaciones de causalidad que explican los factores (endógenos, exógenos y aleatorios) que determinan la evolución de las redes (Snijders et al., 2006). Esto supone un avance sin precedentes en la historia del ARS, puesto que aumenta la capacidad de conocer las variables que determinan el comportamiento de los actores y la nueva configuración de la red que se deriva de estos cambios. Existen diferentes paquetes estadísticos para analizar datos longitudinales como ORA ${ }^{13}$ (Carley et al., 2013), COMMETRIX ${ }^{14}$ (Trier y Bobrik, 2007) y SIENA ${ }^{15}$ (Ripley et al., 2014).

Un segundo tipo de técnica son los modelos de simulación basados en agentes (Macal y North, 2010). Según García-Valdecasas (2011: 91) este tipo de análisis "explica cómo emergen las estructuras sociales a partir de las acciones individuales, y a su vez cómo las creencias, deseos y oportunidades de los individuos son afectadas por dichas estructuras, integrando de este modo el nivel macro y el micro de la realidad social". La simulación es una herramienta de gran utilidad para realizar prospecciones acerca de los cambios que podrían producirse en la estructura de la red, bajo los efectos de determinadas circunstancias que se relacionan con los cambios en el posicionamiento o en el comportamiento de cada actor. En el caso de las redes inter-organizativas, estaríamos hablando de las entidades que participan en la implantación del programa. Aunque es una técnica cuya aplicación en el ámbito del ARS es incipiente, existen algunas aplicaciones como CID-ABM ${ }^{16}$ (O'Madadhain et al., 2005) que efectúan simulaciones con datos relacionales.

Otra de las tendencias en auge, es combinar estrategias de análisis estructural y sistemas de información geográfica (SIG). Aunque el centro de atención de la evaluación estructural son las relaciones que mantienen los actores y la estructura que se deriva de estas relaciones, conviene recordar que estos vínculos se producen en contextos localizados, en los que la proximidad o la lejanía pueden ser factores desencadenantes del establecimiento de relaciones como vimos en el ejemplo mostrado a título ilustrativo en este trabajo. Por tanto es factible pensar que el posicionamiento geográfico es un elemento esencial para comprender los patrones relacionales que se producen en las redes inter-organizativas que implementan programas de intervención en diferentes regiones (i.e., Cardazone et al., 2014). Una de las alternativas es incorporar el posicionamiento geográfico, como un atributo

12 Algunos programas de ARS ofrecen la opción de aislar el conjunto de relaciones que una organización establece dentro de la red. Posteriormente es necesario guardar la matriz de relaciones correspondiente a esta organización y calcular los parámetros de cohesión como haríamos en el caso de redes completas. Sin embargo la comparación de distintas redes egocéntricas, puede exigir normalizar los valores de los indicadores dado que las redes deben tener el mismo número de actores para que los parámetros puedan ser comparables.

13 http://www.casos.cs.cmu.edu/projects/ora/.

14 http://www.commetrix.de/.

15 https://www.stats.ox.ac.uk/ snijders/siena/.

16 http://www.elbirttechnologies.com/software/cid_abm.html. 
-variable- más para caracterizar a los miembros de la red. La combinación de SIG y ARS puede ser una estrategia efectiva para comprender el modo en que la dispersión o la concentración geográfica, determinan la estructura de la red y el rol que desempeñan los actores que la componen.

En última instancia es preciso poner en valor el potencial que nos ofrece la incorporación en el diseño de investigación, de estrategias cualitativas de validación de la información recabada a través de procedimientos cuantitativos. Los estudios que combinan metodología cuantitativa y cualitativa acceden al conocimiento generado a través de la evaluación estructural de un modo comprehensivo. El empleo de estrategias cualitativas hace posible contrastar la información obtenida a través de los indicadores de centralidad y cohesión, adquiriendo un destacado valor interpretativo de estos (Cross et al., 2009). Esto hace posible conocer el contexto en el que se ha generado la información relacional y los factores que determinan la configuración estructural y la posición que ocupan los actores en la red. En este sentido es recomendable incorporar instrumentos propios de la investigación cualitativa, como el grupo de discusión y la entrevista en profundidad. Por ejemplo entrevistar a informantes clave haciendo uso del feedback visual, es una estrategia de gran utilidad para conocer la reacción de los propios actores cuando observan la posición que su organización ocupa en la red. Este tipo de ejercicios consigue poner de manifiesto algunos aspectos que pueden estar definiendo la morfología de la red, y que los investigadores no pueden conocer disponiendo sólo de los indicadores y de los datos capturados a través de cuestionarios.

A modo de cierre queremos expresar algunos desafíos que conlleva la utilización del ARS para evaluar políticas públicas. Un aspecto relevante a tener en consideración son los costes. La obtención y el procesamiento de datos relacionales pueden generar costes elevados, particularmente cuando se obtiene la información a través de cuestionarios o entrevistas personales. Otro elemento a tener en cuenta son las aspectos éticos relativos al uso que se le dará a la información reticular, a la apropiación de la misma por parte del equipo evaluador y al modo en que se garantizará el anonimato y la confidencialidad de las personas que proporcionan los datos (Borgatti y Molina, 2003). A pesar de las limitaciones reseñadas, las herramientas de evaluación estructural pueden ser un gran aliado para los diseñadores y gestores de políticas públicas implementadas a través de coaliciones y redes inter-organizativas.

\section{BIBLIOGRAFÍA}

Abbasi, A., y Kapucu, N. (2012), "Structural dynamics of organizations during the evolution of interorganizational networks in disaster response", Journal of Homeland Security and Emergency Management, 9(1): 1547. DOI: $10.1515 / 1547-7355.1975$.

Atkinson, R., y Flint, J. (2001), “Accessing hidden and hard-to-reach populations: Snowball research strategies”, Social Research Update, 33(1): 1-4.

Bastian, M., Heymann, S., y Jacomy, M. (2009), “Gephi: an open source software for exploring and manipulating networks", ICWSM, 8: 361-362.

Berg, E. (2006), "Snowball sampling-I", Encyclopedia of Statistical Sciences. John Willey \& Sons, Inc. DOI: 10.1002/0471667196.ess2478.pub2.

Biernacki, P., y Waldorf, D. (1981), "Snowball sampling: Problems and techniques of chain referral sampling”, Sociological Methods \& Research, 10(2): 141-163. DOI: 10.1177/004912418101000205.

Borgatti, S.P., y Everett, M.G. (1999), “Models of Core/Periphery structures”, Social Networks, 21: 375-395. DOI: $10.1016 / \mathrm{S} 0378-8733(99) 00019-2$.

Borgatti, S.P., y Molina, J.L. (2003), "Ethical and strategic issues in organizational social network analysis", The Journal of Applied Behavioral Science, 39(3): 337-349. DOI: 10.1177/0021886303258111.

Browne, K. (2005), "Snowball sampling: using social networks to research non-heterosexual women", International Journal of Social Research Methodology, 8(1): 47-60. DOI: 10.1080/1364557032000081663.

Buchthal, O. V., Taniguchi, N., Iskandar, L., y Maddock, J. (2013), “Assessing state-level active living promotion using network analysis", Journal of Physical Activity and Health, 10: 19-32.

Bunger, A. C., Doogan, N. J., y Cao, Y. (2014), “Building Service Delivery Networks: Partnership Evolution Among Children's Behavioral Health Agencies in Response to New Funding”, Journal of the Society for Social Work and Research, 5(4): 513-538. DOI: 10.1086/679224.

Cardazone, G., Sy, A. U., Chik, I., y Corlew, L. K. (2014), “Mapping one strong 'Ohana: Using network analysis and GIS to enhance the effectiveness of a statewide coalition to prevent child abuse and neglect", American Journal of Community Psychology, 53(3-4): 346-356. DOI: 10.1007/s10464-014-9641-7.

Carley, K. M., Pfeffer, J., Reminga, J., Storrick, J., y Columbus, D. (2013), ORA User's Guide 2013. Carnegie Mellon University, School of Computer Science, Institute for Software Research, Technical Report, CMU-ISR-13-108,

Chavis D. (2001), "The Paradoxes and Promise of Community Coalitions", American Journal of Community Psychology, 29(2): 309-320. DOI: 10.1023/A:1010343100379. 
Cross, J. E., Dickmann, E., Newman-Gonchar, R., y Fagan, J. M. (2009), “Using mixed-method design and network analysis to measure development of interagency collaboration", American Journal of Evaluation, 30(3): 310329. DOI: $10.1177 / 1098214009340044$

Dekker, D., Krackhardt, D., y Snijders, T. A. B. (2007), "Sensitivity of MRQAP tests to collinearity and autocorrelation conditions", Psychometrika, 72: 573-581. DOI: 10.1007/s11336-007-9016-1

De Nooy, W., Mrvar, A., y Batagelj, V. (2011), Exploratory social network analysis with Pajek (Vol. 27). Cambridge University Press.

Eisenberg, M., y Swanson, N. (1996), “Organizational network analysis as a tool for program evaluation”, Evaluation \& the Health Professions, 19(4): 488-506. DOI: $10.1177 / 016327879601900407$.

Feinberg, M., Riggs, N. R., y Greenberg, MT (2005), "Social networks and community prevention coalitions", Journal of Primary Prevention, 26(4): 279-298. DOI: 10.1007/s10935-005-5390-4.

Freeman, L. C. (1979), “Centrality in social networks conceptual clarification”, Social Networks, 1(3): 215-239. DOI: 10.1016/0378-8733(78)90021-7.

Friedkin, N. E. (1981), "The development of structure in random networks: an analysis of the effects of increasing network density on five measures of structure”, Social Networks, 3(1): 41-52. DOI: 10.1016/03788733(81)90004-6.

García-Valdecasas, J. I. (2011), “La simulación basada en agentes: una nueva forma de explorar los fenómenos sociales”, Revista Española de Investigaciones Sociológicas, 136(1): 91-109. DOI: 10.5477/cis/reis.136.91.

Gulati, R. (1995), "Social structure and alliance formation patterns: a longitudinal analysis", Administrative Science Quarterly, 40: 619-652. DOI: 10.2307/2393756.

Gulati, R., y Sytch, M. (2007), "Dependence asymmetry and joint dependence in interorganizational relationships: Effects of embeddedness on a manufacturer's performance in procurement relationships", Administrative Science Quarterly, 52(1): 32-69. DOI: 10.2189/asqu.52.1.32.

Haines, V. A., Godley, J., y Hawe, P. (2011), “Understanding interdisciplinary collaborations as social networks”, American Journal of Community Psychology, 47(1-2): 1-11. DOI: 10.1007/s10464-010-9374-1.

Harris, J. K., Luke, D. A., Burke, R. C., y Mueller, N. B. (2008), "Seeing the forest and the trees: using network analysis to develop an organizational blueprint of state tobacco control systems", Social Science \& Medicine, 67(11): 1669-1678. DOI: 10.1016/j.socscimed.2008.07.013.

Harris, J. K., Provan, K. G., Johnson, K. J., y Leischow, S. J. (2012), “Drawbacks and benefits associated with inter-organizational collaboration along the discovery-development-delivery continuum: a cancer research network case study", Implementation Science, 7(1): 69-83. DOI: 10.1186/1748-5908-7-69.

Huisman, M., y van Duijn, Marijtje A. J. (2011), "A reader's guide to SNA software”. En J. Scott and P.J. Carrington (Eds.) The SAGE Handbook of Social Network Analysis (pp. 578-600). London: SAGE.

Huxham, C., y Vangen, S, (2005), Managing to Collaborate: The Theory and Practice of Collaborative Advantage. London: Routledge.

Isett, K. R., Mergel, I. A., LeRoux, K., Mischen, P. A., y Rethemeyer, R. K. (2011), "Networks in public administration scholarship: Understanding where we are and where we need to go", Journal of Public Administration Research and Theory, 21(suppl 1): i157-i173. DOI: 10.1093/jopart/muq061.

Isett, K. R., y Provan, K. G. (2005), "The evolution of dyadic interorganizational relationships in a network of publicly funded nonprofit agencies”, Journal of Public Administration Research and Theory, 15(1): 149-165. DOI: 10.1093/jopart/muioo8.

Jones, C., Hesterly, W. S., y Borgatti, S. P. (1997), "A general theory of network governance: Exchange conditions and social mechanisms", Academy of Management Review, 22(4): 911-945. DOI: 10.5465/AMR.1997.9711022109

Jordana, J. (2009), “Examinando las redes de actores en el análisis de las políticas públicas: debate teórico y técnicas cuantitativas”, Gestión y Análisis de Políticas Públicas, 1: 9-16.

Kickert, Walter J. M., Erik-Hans Klijn, y Koppenjan, J. (eds.) (1997), Managing Complex Networks: Strategies for the Public Sector. London: Sage Publications.

Knoke D., y Yang, S. (2008), Social Network Analysis. Sage Publications Thousand Oacks. California. C. A.

Krackhardt, D. (1988), "Predicting with networks: Nonparametric multiple regression analysis of dyadic data", Social Networks, 10: 359-381. DOI: 10.1016/0378-8733(88)90004-4.

Lecy, J. D., Mergel, I. A., y Schmitz, H. P. (2014), “Networks in Public Administration: Current scholarship in review”, Public Management Review, 16(5): 643-665. DOI: 10.1080/14719037.2012.743577.

Li, J., Weeks, M. R., Borgatti, S. P., Clair, S., y Dickson-Gomez, J. (2012), “A social network approach to demonstrate the diffusion and change process of intervention from peer health advocates to the drug using community", Substance Use \& misuse, 47(5): 474-490. DOI: 10.3109/10826084.2012.644097.

Long, J. C., Cunningham, F. C., y Braithwaite, J. (2013), “Bridges, brokers and boundary spanners in collaborative networks: a systematic review”, BMC Health Services Research, 13(1): 158. DOI:10.1186/1472-6963-13-158. 
Luke, D. A., Harris, J. K., Shelton, S., Allen, P., Carothers, B. J., y Mueller, N. B. (2010), "Systems analysis of collaboration in 5 national tobacco control networks", American Journal of Public Health, 100(7):1290-1297. DOI: $10.2105 / \mathrm{AJPH} .2009 .184358$.

Macal, C. M., y North, M. J. (2010), "Tutorial on agent-based modelling and simulation”, Journal of Simulation, 4(3): 151-162. DOI:10.1057/jos.2010.3.

Mascia, D., Cicchetti, A., y Damiani, G. (2013), “Us and Them: a social network analysis of physicians' professional networks and their attitudes towards EBM", BMC Health Services Research, 13(1): 1-8. DOI: 10.1186/1472-696313-429.

McCarty, C. (2002), "Structure in personal networks", Journal of Social Structure, 3(1). http://www.cmu.edu/joss/ content/articles/volumez/McCarty.html.

Mitchell, J.C. (1969), Social networks in urban settings. Manchester, UK: Manchester University Press.

Mizruchi, M. S., y Marquis, C. (2006), "Egocentric, sociocentric, or dyadic?: Identifying the appropriate level of analysis in the study of organizational networks", Social Networks, 28(3): 187-208. D0I:10.1016/j. socnet.2005.06.002.

O’Madadhain, J., Fisher, D., Smyth, P., White, S., y Boey, Y. B. (2005), “Analysis and visualization of network data using JUNG", Journal of Statistical Software, 10(2): 1-35.

O’Toole, L. J., Jr. (1997), “Treating Networks Seriously: Practical and Research- Based Agendas in Public Administration”, Public Administration Review, 57(1): 45-52. DOI: 10.2307/976691.

Owen-Smith, J., y Powell, W.W. (2004), "Knowledge networks as channels and conduits: The effects of spillovers in the Boston biotechnology community", Organization Science, 15: 5-21. http://dx.doi.org/10.1287/orsc.1030.0054.

Provan, K.G, Fish, A., y Sydow, J. (2007), "Interorganizational Networks at the Network Level: A Review of the Empirical Literature on Whole Networks", Journal of Management, 33(3): 479-516. DOI: 10.1177/0149206307302554.

Provan, K.G., Huang, K., y Milward, B. (2009), "The Evolution of Structural Embeddedness and Organizational Social Outcomes in a Centrally Governed Health and Human Services Network", Journal of Public Administration Research and Theory, 19, 873-893. DOI: 10.1093/jopart/muno36.

Provan, K. G., y Milward, H. B. (2001), "Do networks really work? A framework for evaluating public-sector organizational networks”, Public Administration Review, 61(4): 414-423. DOI: 10.1111/0033-3352.00045.

Provan, K. G., Veazie, M. A., Shaten, L. K., y Teufel-Shone, N. I. (2005), "The Use of Network Analysis to Strengthen Community Partnership", Public Administration Review, 65(5): 603-613. http://www.jstor.org/ stable/3542526.

Ramanadhan, S., et al. (2012), “Addressing cancer disparities via community network mobilization and intersectoral partnerships: a social network analysis”, PloS one, 7(2): e32130. DOI: 10.1371/journal. pone.0032130.

Ramos-Vidal, I. (2015a), “Análisis de Redes Sociales: Una herramienta efectiva para la evaluación de coaliciones comunitarias”, Revista de Salud Pública, 17(3): 323-336. DOI: http://dx.doi.org/10.15446/rsap.v17n3.43051

Ramos-Vidal, I. (2015b), “La detección de actores clave en redes inter-organizativas: una propuesta metodológica”, (Aceptado).

Ramos-Vidal, I. (2015C), “Popularidad y relaciones entre iguales en el aula: Un estudio prospectivo”, Psicología Educativa. (En revisión).

Ramos-Vidal, I., Holgado, D., Maya-Jariego, I., y Palacio, J. (2014)”, Evaluación de procesos comunitarios y análisis de redes inter-organizativas: aportaciones para mejorar la efectividad de las intervenciones comunitarias", Pensando Psicología, 10(17): 135-148. DOI: http://dx.doi.org/10.16925/pe.v10i17.798.

Ramos-Vidal, I., y Maya Jariego, I. (2013), “Alianzas y redes de colaboración entre las agrupaciones culturales de las Artes Escénicas en Andalucía”, Empiria: Revista de Metodología en Ciencias Sociales, 26(2): 15-34. DOI: http://dx.doi.org/10.5944/empiria.26.2013.7151.

Ricaurte, P., y Ramos-Vidal, I. (2015), “Aplicaciones del Análisis de Redes Sociales para investigar entornos digitales", Revista Virtualis, 11(1): 165-194.

Rieder, B. (2013), "Studying Facebook via data extraction: the Netvizz application", Proceedings of the 5 th Annual ACM Web Science Conference (pp. 346-355). ACM.

Ripley, R. M., Snijders, T.A.B., Boda, Zsófia., Vörös, A., y Preciado, P. (2014), Manual for SIENA versión 4.0. Oxford: University of Oxford, Department of Statistics; Nuffield College.

Robins, G., Snijders, T., Wang, P., Handcock, M., y Pattison, P. (2007), "Recent developments in exponential random graph $\left(p^{*}\right)$ models for social networks", Social Networks, 29(2): 192-215. D01:10.1016/j. socnet.2006.08.003.

Rowley, T. J. (1997), "Moving beyond dyadic ties: A network theory of stakeholder influences", Academy of Management Review, 22(4): 887-910. DOI: 10.5465/AMR.1997.9711022107. 
Russell, M. A. (2013), Mining the Social Web: Data Mining Facebook, Twitter, Linkedln, Google+, GitHub, and More. O’Reilly Media, Inc.

Santolaya, F.J., Ramos-Vidal I., Jurado, M.J., Holgado, D., y Maya Jariego, I. (2011), Las condiciones laborales de la Industria Cultural en Andalucía: Redes organizativas y compromiso con la comunidad de los trabajadores de las artes escénicas. Córdoba. Fundación de Estudios Sindicales. Consejería de Cultura Junta de Andalucía. http:// www.estudiossindicales.andalucia.ccoo.es/Documentos/file/Ind_Cult_2011_estudio\%20completo.pdf.

Shelley, G. A., Bernard, H. R., Killworth, P., Johnsen, E., y McCarty, C. (1995), "Who knows your HIV status? What HIV+ patients and their network members know about each other", Social Networks, 17(3): 189-217. DOI:10.1016/0378-8733(95)00262-M.

Snijders, T. A., Pattison, P. E., Robins, G. L., y Handcock, M. S. (2006), “New specifications for exponential random graph models”, Sociological Methodology, 36(1): 99-153. DOI: 10.1111/j.1467-9531.2006.00176.x.

Stufflebeam, D. L., y Shinkfield, A. J. (2007), Evaluation Theory, Models, and Applications. San Francisco CA: JosseyBass.

Tichy, N. M., Tushman, M. L., y Fombrun, C. (1979), “Social network analysis for organizations”, Academy of Management Review, 4(4): 507-519. DOI: 10.5465/AMR.1979.4498309.

Trier, M., y Bobrik, A. (2007), "Analyzing the Dynamics of Community Formation.

using Brokering Activities", En Proceedings of the Third Communities and Technologies Conference (463-477), Michigan. Springer Series. DOI: 10.1007/978-1-84628-905-7_23.

Valente, T. W., Chou, C. P., y Pentz, M. A. (2007), "Community coalitions as a system: Effects of network change on adoption of evidence-based substance abuse prevention", American Journal of Public Health, 97(5): 880886.

Valente, T.W., Coronges, K.A., Stevens, G.D., y Cousineau, M.R. (2008), "Collaboration and competition in a children's health initiative coalition: A network analysis", Evaluation and Program Planning, 31(4): $392-402$. DOI: 10.1016/j.evalprogplan.2008.06.002.

Vedung, E. (2008), Public policy and program evaluation. Transaction Publishers. New Brunswick: New Jersey.

Wasserman, S., y Faust, K. (1994), Social Network Analysis: Methods and Applications. Cambridge MA: Cambridge University Press.

Wendel, M. L., Prochaska, J. D., Clark, H. R., Sackett, S., y Perkins, K. (2010), “Interorganizational network changes among health organizations in the Brazos Valley, Texas", The Journal of Primary Prevention, 31(1-2): 59-68. DOI: 10.1007/s10935-010-0203-y. 


\section{ANEXO I. CUESTIONARIO SOCIO-CÉNTRICO DE UNA RED COMPUESTA POR 30 ORGANIZACIONES}

En este apartado vamos a solicitarte información sobre dos tipos de relaciones que tu organización puede mantener con otros agentes que colaboran en el desarrollo de políticas públicas. En las filas de la siguiente tabla aparecen los nombres de las organizaciones y en las columnas dos tipos de relaciones (intercambio de información sectorial y sobre participación conjunta en proyectos). A continuación debes puntuar el tipo de relación que tu organización mantiene con el resto de componentes:

En la columna A debes puntuar el nivel de intercambio de información en la siguiente escala: $0=$ No se produce intercambio; $1=$ Intercambio esporádico (1 ó 2 veces al año); $2=$ Intercambio eventual (cada 2 o 3 meses); $3=$ Intercambio frecuente (semanalmente).

En la columna B debes indicar si tu organización ha participado en el desarrollo conjunto de alguna actividad productiva en el último año ( $0=$ No; $1=$ Sí).

Importante: Debes dejar en blanco la fila correspondiente a tu organización.

\begin{tabular}{|c|l|l|l|}
\hline N. & $\begin{array}{c}\text { Nombre de la } \\
\text { organización }\end{array}$ & $\begin{array}{c}\text { A } \\
\text { (intercambio de información sobre } \\
\text { actividades sectoriales) }\end{array}$ & (Participación conjunta en proyectos) \\
\hline 1 & & & \\
\hline 2 & & & \\
\hline 3 & & & \\
\hline 4 & & & \\
\hline 5 & & & \\
\hline 6 & & & \\
\hline 7 & & & \\
\hline 8 & & & \\
\hline 9 & & & \\
\hline 10 & & & \\
\hline 11 & & & \\
\hline 12 & & & \\
\hline 13 & & & \\
\hline 14 & & & \\
\hline 15 & & & \\
\hline 16 & & & \\
\hline 17 & & & \\
\hline 18 & & & \\
\hline 19 & & & \\
\hline 20 & & & \\
\hline 21 & & & \\
\hline 22 & & & \\
\hline 23 & & & \\
\hline 24 & & & \\
\hline 25 & & & \\
\hline 26 & & & \\
\hline 27 & & & \\
\hline 28 & & & \\
\hline 29 & & & \\
\hline 30 & & & \\
\hline
\end{tabular}




\section{ANEXO II. EJEMPLO DE CUESTIONARIO EGOCÉNTRICO DE LA RED DE UNA ORGANIZACIÓN}

En este apartado vamos a solicitarte información sobre diferentes tipos de relaciones que tu organización puede mantener con otros agentes que colaboran en el desarrollo de políticas públicas. Para ello te vamos a pedir que nos indiques el nombre de otras organizaciones con las que tu entidad colabora en la implementación de políticas públicas o de programas relacionados con las mismas.

A. ¿Con qué organizaciones intercambias información de interés para el desarrollo del programa?

B. ¿A qué organizaciones has solicitado ayuda material para desarrollar un programa?

C. ¿Con qué organizaciones has colaborado en el diseño de un proyecto?

D. ¿Con qué organización te gustaría mantener contacto en un futuro para desarrollar una acción vinculada a la implantación de una política pública?

\begin{tabular}{|c|c|c|c|c|c|}
\hline N. $0^{\circ}$ & Nombre de la organización & A & B & C & D \\
\hline 1 & & $\square$ & $\square$ & $\square$ & $\square$ \\
\hline 2 & & $\square$ & $\square$ & $\square$ & $\square$ \\
\hline 3 & & $\square$ & $\square$ & $\square$ & $\square$ \\
\hline 4 & & $\square$ & $\square$ & $\square$ & $\square$ \\
\hline 5 & & $\square$ & $\square$ & $\square$ & $\square$ \\
\hline 6 & & $\square$ & $\square$ & $\square$ & $\square$ \\
\hline 7 & & $\square$ & $\square$ & $\square$ & $\square$ \\
\hline 8 & & $\square$ & $\square$ & $\square$ & $\square$ \\
\hline 9 & & $\square$ & $\square$ & $\square$ & $\square$ \\
\hline 10 & & $\square$ & $\square$ & $\square$ & $\square$ \\
\hline 11 & & $\square$ & $\square$ & $\square$ & $\square$ \\
\hline 12 & & $\square$ & $\square$ & $\square$ & $\square$ \\
\hline 13 & & $\square$ & $\square$ & $\square$ & $\square$ \\
\hline 14 & & $\square$ & $\square$ & $\square$ & $\square$ \\
\hline 15 & & $\square$ & $\square$ & $\square$ & $\square$ \\
\hline 16 & & $\square$ & $\square$ & $\square$ & $\square$ \\
\hline 17 & & $\square$ & $\square$ & $\square$ & $\square$ \\
\hline 18 & & $\square$ & $\square$ & $\square$ & $\square$ \\
\hline 19 & & $\square$ & $\square$ & $\square$ & $\square$ \\
\hline 20 & & $\square$ & $\square$ & $\square$ & $\square$ \\
\hline 21 & & $\square$ & $\square$ & $\square$ & $\square$ \\
\hline 22 & & $\square$ & $\square$ & $\square$ & $\square$ \\
\hline 23 & & $\square$ & $\square$ & $\square$ & $\square$ \\
\hline 24 & & $\square$ & $\square$ & $\square$ & $\square$ \\
\hline 25 & & $\square$ & $\square$ & $\square$ & $\square$ \\
\hline 26 & & $\square$ & $\square$ & $\square$ & $\square$ \\
\hline 27 & & $\square$ & $\square$ & $\square$ & $\square$ \\
\hline 28 & & $\square$ & $\square$ & $\square$ & $\square$ \\
\hline 29 & & $\square$ & $\square$ & $\square$ & $\square$ \\
\hline 30 & & $\square$ & $\square$ & $\square$ & $\square$ \\
\hline
\end{tabular}

